\title{
ARTICLE Quitting starts in the brain: a randomized controlled trial of app-based mindfulness shows decreases in neural responses to smoking cues that predict reductions in smoking
}

\author{
Amy C. Janes ${ }^{1,2}$, Michael Datko ${ }^{3,4}$, Alexandra Roy ${ }^{5}$, Bruce Barton ${ }^{6}$, Susan Druker ${ }^{6}$, Carolyn Neal ${ }^{7}$, Kyoko Ohashi ${ }^{1,2}$, Hanif Benoit ${ }^{6}$,
} Remko van Lutterveld ${ }^{5}$ and Judson A. Brewer ${ }^{5}$

Current treatments for smoking yield suboptimal outcomes, partly because of an inability to reduce cue-induced smoking. Mindfulness training (MT) has shown preliminary efficacy for smoking cessation, yet its neurobiological target remains unknown. Our prior work with nonsmokers indicates that MT reduces posterior cingulate cortex (PCC) activity. In individuals who smoke, the PCC, consistently a main hub of the "default mode network," activates in response to smoking cues. In this randomized controlled trial, we tested the effects of app-delivered MT on PCC reactivity to smoking cues and whether individual differences in MTmediated PCC changes predicted smoking outcomes. Smoking cue-induced PCC reactivity was measured using functional magnetic resonance imaging at baseline and 1 month after receiving smartphone app-based MT ( $n=33$ ) vs. an active control (National Cancer Institute's QuitGuide, $n=34$ ). Whether individual differences in treatment-related changes in PCC activity predicted smoking behavior was assessed. The MT group demonstrated a significant correlation between a reduction in PCC reactivity to smoking cues and a decline in cigarette consumption $(r=0.39, p=0.02)$. No association was found in the control group $(r=0.08, p=0.65$ ). No effects of group alone were found in PCC or cigarette reduction. Post hoc analysis revealed this association is sex specific (women, $r=0.49, p=0.03$; men: $r=-0.08, p=0.79$ ). This initial report indicates that MT specifically reduces smoking cue-induced PCC activity in a subject-specific manner, and the reduction in PCC activity predicts a concurrent decline in smoking. These findings link the hypothesized behavioral effects of MT for smoking to neural mechanisms particularly in women. This lays the groundwork for identifying individuals who may benefit from targeted digital therapeutic treatments such as smartphone-based MT, yielding improved clinical outcomes.

Neuropsychopharmacology (2019) 44:1631-1638; https://doi.org/10.1038/s41386-019-0403-y

\section{INTRODUCTION}

Nicotine dependence is a chronic relapsing condition. Although $>70 \%$ of smokers want to quit, fewer than $5 \%$ achieve abstinence annually [1]. Failure is related to several factors, including withdrawal (for review see [2]) and smoking-related cue exposure [3]. Smoking cues are known to motivate behavior: seeing someone else smoke elicits strong urges [4] and exposure to smoking cues predicts craving and smoking [5]. The ubiquitous nature of, and exposure to, smoking-related cues provides a major barrier to quitting [6]. Thus, reducing reactivity to smoking cues may be a promising strategy vs. cue avoidance or other approaches. Therefore, it is important to test strategies that attenuate the neurobiological mechanisms involved in cue reactivity, as such brain changes may inform and enhance smoking cessation treatment [7].

Although a number of brain regions have been implicated in cigarette cue-reactivity, the posterior cingulate cortex (PCC), a major node of the default mode network (DMN), has come into focus. As part of the DMN, the PCC has been linked to selfreference, such as when someone is thinking about themselves, planning future events, or daydreaming [8-12]. Additionally, PCC activation has been linked to cue-reactivity across addictive disorders, including smoking [13, 14], cocaine [15], and pathological gambling [16]. Critically, a recent meta-analysis found that smoking cues reliably evoked PCC reactivity [7], while a case study showed that a lesion to the PCC led to quitting [17].

Increased PCC activity has been suggested to be associated with "getting caught up" in one's experience, such as getting fixated on a particular thought, lost in mind-wandering, or pulled into drug craving [18-20]. Conversely, the PCC becomes deactivated during non-self-referential tasks, such as being focused on a task or during mindfulness meditation [20, 21]. In fact, several cross-sectional studies of nonsmokers have found

\footnotetext{
${ }^{1}$ McLean Imaging Center, McLean Hospital, Belmont, MA 02478, USA; ${ }^{2}$ Harvard Medical School, Boston, MA 02115, USA; ${ }^{3}$ Martinos Center for Biomedical Imaging, Department of Radiology, Massachusetts General Hospital, 149 Thirteenth St. \#2301, Charlestown, MA 02129, USA; ${ }^{4}$ Center for Mindfulness and Compassion, Cambridge Health Alliance, 1035 Cambridge St. \#21, Cambridge, MA 02141, USA; ${ }^{5}$ Mindfulness Center, Brown University School of Public Health and Warren Alpert School of Medicine, 121 S Main St, Providence, RI 02903, USA; ${ }^{6}$ Department of Population and Quantitative Health Sciences, University of Massachusetts Medical School, 55 Lake Ave North, Worcester, MA 01655, USA and ${ }^{7}$ University of Oklahoma-Tulsa School of Community Medicine, Tulsa, OK 74135, USA Correspondence: Judson A. Brewer (judson_brewer@brown.edu)

Clinical Trials registration: Mechanisms of Mindfulness for Smoking Cessation; URL_https://clinicaltrials.gov/ct2/show/NCT02943499?term=NCT02943499\&rank=1; Registration Number-NCT02943499
}

Received: 7 February 2019 Revised: 19 April 2019 Accepted: 22 April 2019

Published online: 30 April 2019 
that engaging in mindfulness decreases PCC activity in experienced meditators compared to novices across three different meditation techniques [20, 22]. Further, the PCC deactivated during focused attention in a meta-analysis of 78 studies of meditation [23]. This prior work in nonsmokers suggests that in a smoking population, mindfulness may impact the PCC in a manner that is opposite to the effects of smoking cues. These prior results also fit with the purported role of the PCC as a hub of the DMN, as well as the theoretical underpinnings of mindfulness: selfreferential processing may represent getting caught up in one's internal experience, while mindfulness trains the individual to "let go." In other words, we hypothesize that individuals who learn to be mindful of cravings may be more able to avoid getting caught up in them, with a concomitant reduction in PCC activation [9]. Together, these studies provide a specific brain-based mechanistic model of how mindfulness changes habitual behaviors [24, 25].

Mindfulness has been operationalized to include complementary components of present-centered awareness and acceptance. Thus, mindfulness training (MT) teaches individuals to work with triggers and cue-induced affective states as they arise, rather than reacting to urges by smoking [26]. Mindfulness-based interventions, such as Mindfulness-Based Relapse Prevention, demonstrate some evidence for reduced substance use (reviewed in [27]) $[28,29]$. With regard to smoking, MT showed greater cessation in smoking compared to cognitive therapy (31 vs. 6\%) [30]. Further, MT moderated a decoupling of cue-induced craving and smoking, suggesting an extinction of operantly conditioned habits [26, 31].

App-based smartphone interventions are increasingly used to deliver behavioral treatments because of their relative low cost and enhanced accessibility. This new class of "digital therapeutics" may be an effective way to deliver MT, as just-in-time interventions can be accessed both in context and on demand when triggered by cues. Additionally, app-based delivery affords unique research advantages when studying the neural mechanisms of mindfulness, as it maintains $100 \%$ fidelity, and the "dose" of training can be accurately tracked.

An important next step in understanding the mechanisms of mindfulness is determining whether cue-induced PCC reactivity is decreased by app-based MT, and how such a change in specific brain activity relates to smoking. Further, the field is calling for next steps in capitalizing on individual variability as a way to move beyond group-level effects. For example, recent studies have leveraged individual differences to improve diagnostic power and determine level of severity in substance use disorders [14, 32, 33]. With previously identified target regions of interest, these methods can now be applied to identifying mechanisms of mindfulness at the level of the individual.

In this study, we examined the neurobiological effects of appdelivered MT on smoking cue-reactivity in the PCC (our a priori defined region of interest), and whether changes therein predicted smoking reduction at the level of the individual. We hypothesized that smokers who engaged in MT would demonstrate reductions in PCC cue-reactivity and that these reductions would predict reductions in cigarette smoking at the level of the individual.

\section{MATERIALS AND METHODS}

Study overview and timeline

This was a parallel-group study with equal randomization and individuals were recruited using local flyers, and Facebook ads. The project director screened participants and eligibility criteria included: (1) smoking $>10$ cigarettes/day; (2) $<3$ months of smoking abstinence in the previous year; (3) $8+$ of 10 on a "Readiness-to-change" scale [34]; (4) owning a smartphone; and (5) age between 21 and 65. Participants were excluded if they reported (1) a history of serious neurological or psychiatric conditions (beyond nicotine dependence; e.g. seizure disorder, history of a stroke, schizophrenia, bipolar disorder etc.); (2) changing dose of any psychoactive medication in the past 3 months; (3) previous experience with mindfulness-based stress reduction or equivalent; (4) current meditation or yoga practice (>30 min/day for >5 days); (5) current alcohol abuse; (6) claustrophobia; (7) pregnancy; (8) MRI incompatibility; or (9) tested positive for illegal psychoactive substances or cannabis. All participants underwent informed consent procedures prior to participation, and their participation was voluntary. Participants were given $\$ 25$ upon completion of the first functional magnetic resonance imaging (fMRI) visit and $\$ 75$ upon completion of the second visit. This study was approved by the Institutional Review Board of the University of Massachusetts Medical School (UMMS).

At baseline, eligible participants performed a smoking cuereactivity $\mathrm{fMRI}$ task at UMMS. They were then given a sealed envelope with their random assignment: an app-based MT or active control app. This was generated in variable blocks of four and six by an independent statistician and the envelopes were prepared by an individual independent of data acquisition. An experimenter helped install the assigned app on their smartphone immediately following the first MRI visit, while making sure the participant understood the features of the app and could demonstrate how to use it. Participants were instructed to use their app to help them quit smoking over the next 4-week period. At the posttreatment visit, participants completed the same cuereactivity task as baseline. Smoking status was verified with a carbon monoxide breathalyzer test at each visit.

\section{Smartphone-based app interventions}

The app-based MT program is a phone app designed to deliver core elements of a manualized MT program for smoking cessation with high fidelity $[30,35]$. The program has 22 unique learning modules (5-15 min/module) consisting of daily training videos and in vivo on-demand exercises. Program features are designed to help users self-monitor their smoking habits, identify triggers for smoking, learn methods to become more aware of cravings and use mindfulness practices to ride them out (see Supplementary Text S1). The program calculates and encourages a gradual taper over a 3-week period based on baseline cigarette use.

Participants in the control group used the National Cancer Institute's QuitGuide app (NCI) (https://smokefree.gov/tools-tips/ apps/quitguide). $\mathrm{NCl}$ is based on the design and principles of Smokefree.gov, the most accessed smoking cessation website in the US. It includes strategies for quitting and health outcomes information.

\section{Expectancy evaluation}

Expectancy was assessed at baseline (please refer to Supplementary Text S2 for the questions) [36]. Post treatment, participants were asked, "How likely are you to recommend this app to a friend?"

\section{Study outcomes}

The primary outcome of this study was to demonstrate that appbased MT reliably decreased PCC reactivity to smoking cues.

\section{Functional MRI task}

At each MRI visit, participants completed a previously developed cue-reactivity task (Fig. 1) [13, 37]. To reduce variability related to cravings during the MRI task, participants smoked immediately prior to scanning. Participants were shown 60 smoking, 60 neutral, and 10 target images divided evenly across 5 scanning blocks lasting $5 \mathrm{~m} 18 \mathrm{~s}$ each, for a total task time of $26.5 \mathrm{~min}$. Images were presented for $4 \mathrm{~s}$ each in a pseudorandom order. Participants were shown a fixation cross on a black screen during jittered inter-trialintervals, which ranged from 6 to $14 \mathrm{~s}$ ( $10 \mathrm{~s}$ average). Smoking images included smoking-related content such as people smoking or holding cigarettes. Neutral images were matched for content. 


\section{Pre-treatment Cue Reactivity}
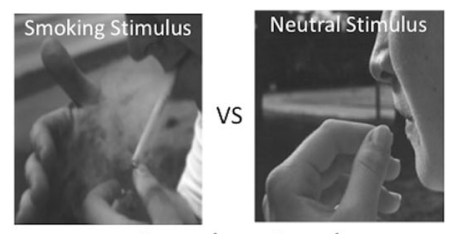

Randomized

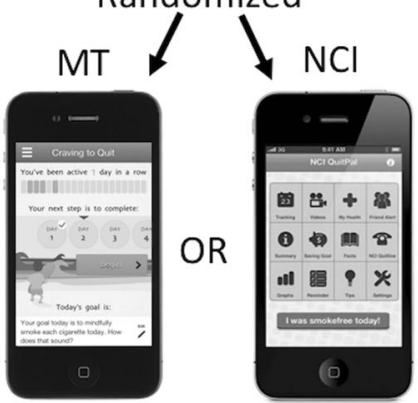

Post-treatment Cue Reactivity
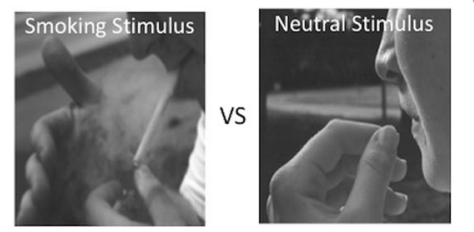

PCC Reactivity:

Pre vs. Post

Intervention

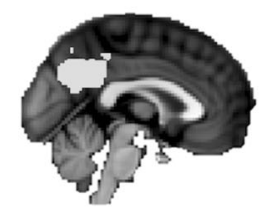

Follow up $\mathrm{fMRI}$

Scan

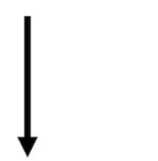

Analysis

Fig. 1 Study design. Yellow overlay on the anatomic brain image at the bottom represents the a priori defined PCC ROI derived from Janes et al. [37, 39] for this analysis. PCC posterior cingulate cortex, $\mathrm{ROI}$ region of interest

All images were novel in that no image shown on the preintervention visit was repeated during the second scan. Task instructions were to pay attention to all images, but to respond with a button-press any time they saw an image of an animal. Animals were used as target images to ensure participants stayed awake and attended to the task, but were not included in analyses. Between each of the five task scans, participants were asked whether they had fallen asleep at any point during the scan and it was repeated if the participant reported falling asleep $(n=$ 2, baseline).

Neuroimaging data collection

Imaging data were collected on two Philips 3 Tesla MRI scanners (see Supplementary Fig. S1, which showed no significant differences between scanners). High-resolution anatomical MRI scans were acquired using the MPRAGE protocol with the following parameters: 181 (sagittal) slices, repetition time (TR)/ echo time (TE): $7.0 / 3.2 \mathrm{~ms}$, shot interval: $3000 \mathrm{~ms}$, field of view (FOV) $240 \times 240 \times 181$, matrix $=240 \times 240 ; 1 \mathrm{~mm}$ isotropic voxels. Cue-reactivity task scans were collected with the following parameters: 139 scans, 37 (transverse) slices; TR/TE: 2000/30 ms, FOV $216 \times 216 \times 130$, matrix $=80 \times 80 ; 2.7 \times 2.7 \times 3.5 \mathrm{~mm}$ voxels.

fMRI preprocessing

fMRI data analysis was conducted using tools from the fMRI of the Brain Software Library (FSL; www.fmrib.ox.ac.uk/fsl). The first five volumes for each run were removed to allow for signal stabilization. Functional data were preprocessed using the following: (1) motion correction with MCFLIRT, (2) brain extraction using BET, (3) slice timing correction, (4) spatial smoothing with a Gaussian kernel of full-width half-maximum $6 \mathrm{~mm}$, and (5) highpass temporal filter with Gaussian-weighted least-squares straightline fitting with $\sigma=100 \mathrm{~s}$. A script was also used to detect and adjust for artifacts due to motion and intensity spiking prior to conducting preprocessing within FSL [38]. Participants' structural images were registered to the MNI152 $2 \mathrm{~mm}$ standard space template; corresponding functional datasets were transformed into standard space at $2 \mathrm{~mm}^{3}$ resolution using the resulting registration transformation matrices.

Cue-reactivity analysis

First-level analysis was conducted on each of the participant's five cue-reactivity runs separately. First-level general linear model included three regressors, convolved with the gamma hemodynamic response function, corresponding to smoking, neutral, and target image presentation. Confound regressors based on rigidbody head-motion parameters were included to model motion effects. Consistent with our prior work [37, 39], another regressor was included that represented motion/intensity artifacts identified and removed prior to preprocessing. Contrasts between smoking and neutral image conditions were created for each run. First-level results were then combined across task scans using a second-level fixed effects analysis to obtain the average contrast-related brain reactivity for each participant. To determine the Group by Treatment interaction on PCC reactivity to smoking $>$ neutral cues, a repeated measures ANOVA was conducted on betaweights for smoking $>$ neutral contrasts that were extracted from the PCC region-of-interest (ROI), which was based on an independent dataset evaluating the smoking $>$ neutral contrast at the whole brain level (Fig. 1, bottom) [37]. To examine betweengroup differences in pre- vs. posttreatment cue reactivity, the posttreatment PCC BOLD response for the smoking vs. neutral contrast was subtracted from the baseline response. The groups were then compared directly on this measure using a $t$ test.

To confirm that the task activated the PCC at baseline, a grouplevel voxel-wise analysis was conducted, restricting the analysis to the PCC. Cluster-level correction was applied using randomize, FSL's tool for nonparametric permutation inference, $z=3.1, p<0.05$.

Study blinding

Participants were blinded to group and team membership. Team members who randomized participants and performed scans did not perform study analyses. The principal investigator and team members who conducted fMRI and statistical analysis were pseudo-blinded to group until all analyses were complete.

Sample size

Our prior work with experienced meditators showed a percent signal change in PCC activity of $-0.3(S D=0.2)$, while novices showed a percent signal change of $0.0(S D=0.2)$ [20], which yielded an effect size (ES) of 1.5. To be conservative we used an ES of 0.9 for power calculation; a sample size of 20 subjects/group provided $80 \%$ power to detect an ES of 0.9 with two-sided $5 \%$ type I error using a two-sample $t$ test.

Correlation analysis

The relationship between treatment-induced changes in PCC reactivity to smoking $>$ neutral cues and the change in number of cigarettes smoked per day was evaluated using Spearman's correlation coefficient. Correlation comparison was performed using a standard $z$ test of Fisher's z-transformed correlations. Cigarette use change scores and change in PCC reactivity $(\Delta)$ were calculated by subtracting baseline from posttreatment values. To 
Table 1. Baseline demographic characteristics

\begin{tabular}{|c|c|c|c|}
\hline & MT $(N=33)$ & $\mathrm{NCl}(N=34)$ & $p$ value \\
\hline Age (mean with standard deviation in parentheses) & $46(11)$ & $43(11)$ & 0.20 \\
\hline $\begin{array}{l}\text { Work status (full-time/part-time/homemaker (not looking for job)/disabled-unable } \\
\text { to work/retired/unemployed) }\end{array}$ & $24 / 3 / 0 / 0 / 3 / 3$ & $25 / 4 / 1 / 0 / 0 / 4$ & 0.43 \\
\hline Number of cigarettes at baseline (mean with standard deviation in parentheses) & $17(6)$ & $16(6)$ & 0.79 \\
\hline
\end{tabular}

examine the role of app modules in the change in smoking, a linear regression model was created for each group with change in smoking as the dependent variable and, as independent variables, $\triangle \mathrm{PCC}$, the number of modules completed, and the baseline number of cigarettes smoked. To determine whether effects were specific to the PCC, the medial prefrontal cortex ( $\mathrm{mPFC}$ ) and bilateral anterior insula (dAl) were evaluated. The mPFC ( $8 \mathrm{~mm}$ sphere centered on $x=-4, y=54, z=4$ ) was chosen given that it typically coactivates with the PCC to smoking cues and shows decreased activation in experienced meditators [20]. In contrast, the dAI ROI, which has been used in our prior work [37] does not show decreased activation in meditators, yet has been linked to nicotine dependence.

\section{RESULTS}

Participants

Eighty-three participants (average age $=44.4$ years) were recruited (see Table 1) between December 2016 and July 2018, of which 67 participants completed the study and were used in the final analysis (see CONSORT diagram, Fig. 2). One participant was excluded because of concussive symptoms unrelated to the study. No participants reported any type of current meditation or yoga practice at the time of screening.

\section{Behavioral and app data}

There were no differences in app uses between the MT (completed an average of $15.6 \pm 6.9$ out of 22 modules), and $\mathrm{NCl}$ groups (completed $15.6 \pm 6.6$ modules, see Table 2, $p=0.93$, $d=0)$. At baseline, individuals in the MT group rated the usefulness of the app as a $6.7 \pm 1.8$ out of 9 , their confidence in recommending the app to a friend as $5.6 \pm 2.3$ out of 9 , and their likelihood to quit as $81 \pm 19 \%$ out of $100 \%$. Individuals in the $\mathrm{NCl}$ group rated the usefulness of the app as a $6.9 \pm 1.8$ out of 9 , their confidence in recommending the app to a friend as $5.8 \pm 2.0$ out of 9 , and their likelihood to quit as $80 \pm 19 \%$ out of $100 \%$. No differences in these expectancies were found between the groups (see Supplementary Table, S1). At follow-up, the MT group reported a significantly higher likelihood of recommending to a friend (9.6 \pm 0.7 vs. $5.9 \pm 3.4, p<0.001, d=1.5$ ).

At baseline, individuals reported smoking $16.8 \pm 5.5$ (MT) and $16.6 \pm 5.9(\mathrm{NCl})$ cigarettes/day. At post intervention they reported smoking $5.4 \pm 5.6(\mathrm{MT})$ and $7.9 \pm 8.0(\mathrm{NCl})$ cigarettes/day, for an average reduction of $11.4 \pm 7.4(p<0.0001, d=2.05)$ in the MT group and $8.7 \pm 5.8(p<0.0001, d=1.28)$ cigarettes/day in the $\mathrm{NCl}$ group (Table 2). At baseline, participants who completed all procedures $(n=67)$ were not statistically different from those who dropped out $(n=16)$.

A significant correlation between the number of training modules that an individual completed and the change in number of cigarettes smoked/day was found in the MT group $\left(r_{\mathrm{s}}=0.49\right.$, $p=0.004)$, but not in the $\mathrm{NCl}$ group $\left(r_{\mathrm{s}}=0.20, p=0.25\right)$.
Brain reactivity to smoking $>$ neutral cues and correlations with smoking outcomes

Subject motion was low with no difference in absolute mean displacement between groups on either the baseline $(p=0.45$, $d=0.19, \mathrm{MT}=0.18 \mathrm{~mm}, \mathrm{NCl}=0.19 \mathrm{~mm}$ ) or the posttreatment visit $(p=0.41, d=0.20, \mathrm{MT}=0.20 \mathrm{~mm}, \mathrm{NCl}=0.22 \mathrm{~mm}$ ).

Both groups demonstrated significant PCC cue reactivity at baseline (Supplementary Fig. S2, $z=3.1, p<0.05$, corrected using nonparametric permutation testing). A repeated measures ANOVA revealed no main effect of Visit (baseline/post-treatment), Group $(\mathrm{MT} / \mathrm{NCl})$ or interaction between the two in pre- to posttreatment PCC cue reactivity to smoking $>$ neutral cues (Table 2 ).

No between-group difference in pre- vs. posttreatment PCC cue-reactivity was found when the differences were directly compared (MT: $-3.6 \pm 33.9,95 \% \mathrm{Cl}=12.0 ; \mathrm{NCl}:-2.77 \pm 33.0,95 \%$ $\mathrm{Cl}=11.5 ; p=0.92, d=0.02$ ).

A significant correlation was found between the $\triangle \mathrm{PCC}$ score and the change in number of cigarettes smoked/day in the MT group ( $n=33, r_{\mathrm{s}}=0.39, p=0.02$, Fig. 3 ) but not in the $\mathrm{NCl}$ group $\left(n=34, r_{\mathrm{s}}=0.08, p=0.65\right)$. Post-hoc analyses of the control regions indicated that the change in number of cigarettes in the MT group was also correlated with the reduction in $\mathrm{mPFC}$ activation $\left(r_{\mathrm{s}}=0.35, p=0.05\right)$, but not within the bilateral dAl $\left(r_{\mathrm{s}}=-0.08, p=0.64\right)$.

To investigate the joint association of $\triangle P C C$ and number of modules viewed on the change in cigarette smoking, separate linear regression models were fitted for each group. In the linear regression model combining $\triangle P C C$, number of modules completed, and cigarettes smoked at baseline, all predictors were significantly related to the change in cigarette smoking for the MT group: change in PCC (coefficient $=0.07 ; \quad t$-statistic $=2.55$; $p=0.02$ ), number of modules (coefficient $=0.30 ; t$-statistic $=$ 2.28; $p=0.03$ ), and baseline number of cigarettes smoked (coefficient $=0.75 ; t$-statistic $=4.53 ; p<0.0001$ ). This indicates that there was a decrease of $0.07,0.30$, and 0.75 cigarettes/day smoked for every unit decrease in the PCC, every module viewed, and every cigarette smoked at baseline, respectively. The $R^{2}$ for this model was 0.58 . Conversely, for the $\mathrm{NCl}$ group, no predictors were associated with changes in cigarette smoking, all $p$ values $\geq 0.20$. The $R^{2}$ for this model was 0.08 .

A post-hoc evaluation of sex revealed that the $\triangle \mathrm{PCC}$ correlation with the change in number of cigarettes smoked/day was significant in females receiving MT $\left(n=20, r_{\mathrm{s}}=0.49, p=0.03\right)$, but not in males $\left(n=13, r_{\mathrm{s}}=-0.08, p=0.79\right.$ ) (see Supplementary Fig. S3). This between-sex difference was significant $(z=1.7, p=$ 0.04 , one-tailed). No association was noted in the $\mathrm{NCl}$ group even when evaluating females $\left(n=24, r_{\mathrm{s}}=-0.06, p=0.78\right)$ and males ( $n=9, r_{\mathrm{s}}=0.48, p=0.19$ ) independently.

Post-hoc analysis revealed that at baseline $13.4 \%$ of subjects did not demonstrate any PCC cue reactivity (smoking $>$ neutral beta weights $>0$ ). A chi square test revealed no differences between groups in the number of nonreactive individuals at baseline 


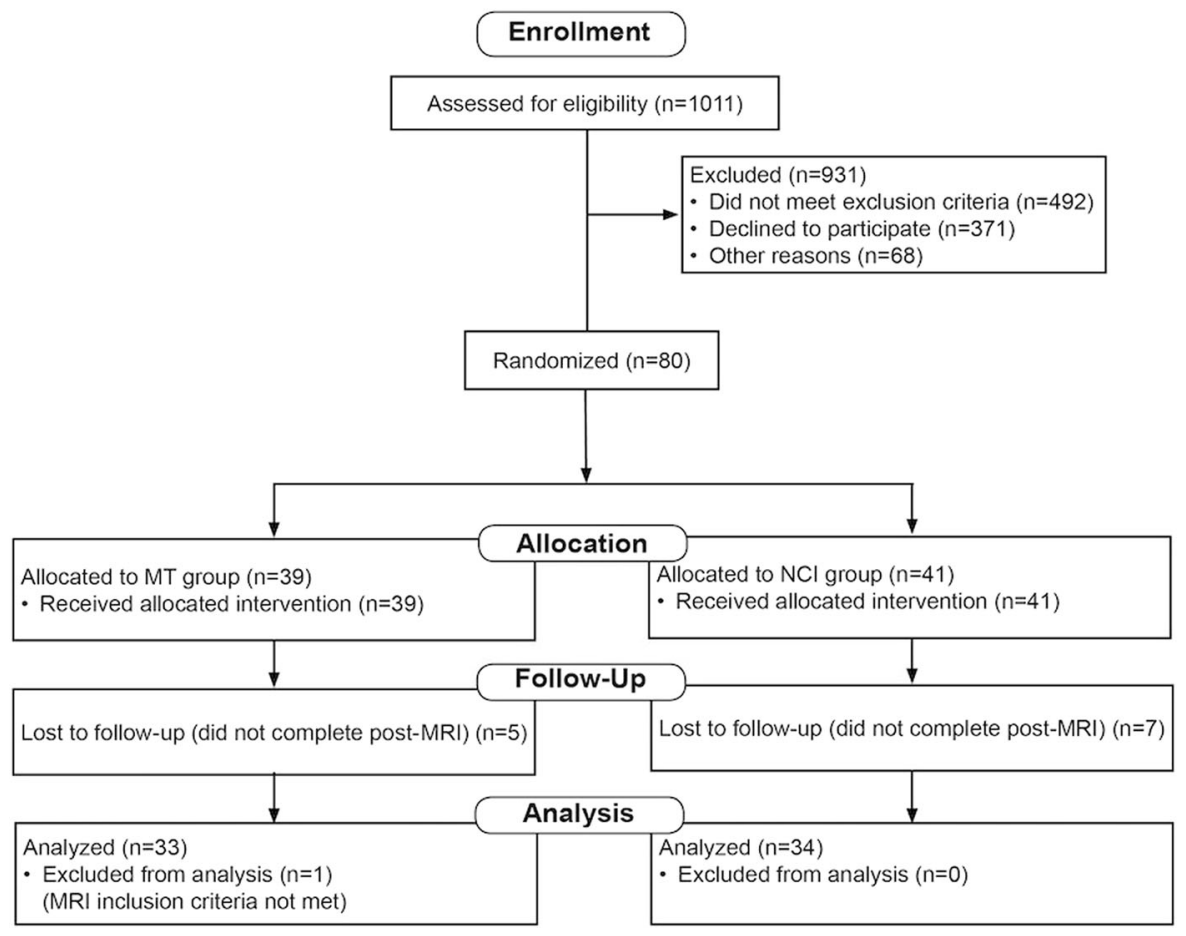

\section{Fig. 2 CONSORT diagram}

$\left(x^{2}=1.02, p=0.32\right)$ or at posttreatment $\left(x^{2}=0.54, p=0.46\right)$. In the MT group, cue-reactive individuals showed significantly higher reduction in cigarette use than nonreactive individuals $(p<.005$, $d=1.47)$, whereas no difference was found in the $\mathrm{NCl}$ group $(p=$ $0.99, d=-0.003$ ). At a group level, individuals who received the MT intervention who showed a reduction in cue-reactivity from pre- to posttreatment also demonstrated significantly greater reduction in cigarettes smoked than those who showed increased cue-reactivity (reduction of 14.12 vs. 8.56, $p=0.02, d=0.79$ ), whereas no differences were found in the $\mathrm{NCl}$ group (reduction of 9.63 vs. $7.47, p=0.23, d=0.37$ ). No adverse events related to either of the study interventions were reported through active monitoring.

\section{DISCUSSION}

The current work is the first to study the neural mechanisms of MT on smoking cue-reactivity at the level of the individual. Using a novel app-based delivery modality, we found that a reduction in cue-induced PCC reactivity predicted a concomitant reduction in cigarette smoking, an effect that was specific to individuals in the MT group. An association was also found between the reduction in cigarettes smoked/day and the number of modules of MT completed (a proxy for "dose") as well as a joint association between completed modules and $\triangle P C C$ activity, which together with the number of cigarettes smoked at baseline accounted for $58 \%$ of the variance; these effects were not observed in the $\mathrm{NCl}$ group, suggesting specificity far beyond expectancy, motivation and the general use of an appbased smoking cessation treatment modality. Post-hoc analysis revealed that this effect was largely driven by women. An alternate interpretation for a lack of correlation seen in the $\mathrm{NCl}$ group is that its effects were found regardless of the amount of times that the app was used.

While the $\mathrm{NCl}$ program provides generalized cessation support, MT provides a focused strategy that specifically trains individuals to reduce how "caught up" they get by cue-induced thoughts/ emotions and resultant smoking. In line with previous studies demonstrating PCC cue-reactivity in smokers, and reductions in PCC activity during meditation in nonaddicted populations, these results are the first to support the hypothesized neurobiological mechanism underlying the behavioral effectiveness of mindfulness in smoking cessation in that they demonstrate a specific effect of MT (reduced PCC reactivity) predicting a clinically relevant outcome (a reduction in cigarette smoking) at the level of the individual.

The impact of MT was not specific to the PCC, but also included the mPFC. Collectively, these functionally connected regions are primary nodes of the DMN [8] and typically coactivate in response to smoking cues [37] while also showing concurrent deactivation in experienced meditators [20]. Thus, it was unsurprising that MT had a similar impact in both regions. However, MT's influence was not nonspecific, as an effect was not found in the $d A l$, a brain region that has been strongly linked with nicotine dependence [37].

Consistent with prior work, at baseline, the PCC showed significant activation to smoking $>$ neutral cues in both groups [7, 37-39]. Importantly, reductions in PCC reactivity, at a group level, were not specific enough to predict outcomes; only when individual variability was taken into account did the modeling have sufficient granularity to yield specific effects on smoking. This may be in part due to the MT helping some individuals more than others not get caught up in craving (and concomitant PCC reactivity). Previous studies have shown that PCC reactivity is increased during smoking abstinence [40], even when pharmacological aides are utilized [41]. Our data demonstrated a dichotomy in smoking outcomes that was specific to MT: individuals who reduced PCC activity from pre-post showed a significantly greater reduction than those who increased PCC activity (no effect was found in the $\mathrm{NCl}$ group). Further supporting the importance of accounting for individual differences in studies such as this one, individuals in the MT group who lacked baseline PCC cue-reactivity showed a significantly attenuated reduction in cigarette smoking. Those lacking baseline PCC cue-reactivity may not be as "caught up" by cue-induced craving and therefore may be less likely to need or to behaviorally respond to MT, but instead may require other cessation aids addressing their specific reasons for smoking. 
Table 2. Brain and behavioral changes with app-based training. Combined and group-wise results for smoking cue reactivity in PCC, cigarettes smoked per day, and number of app modules completed are reported

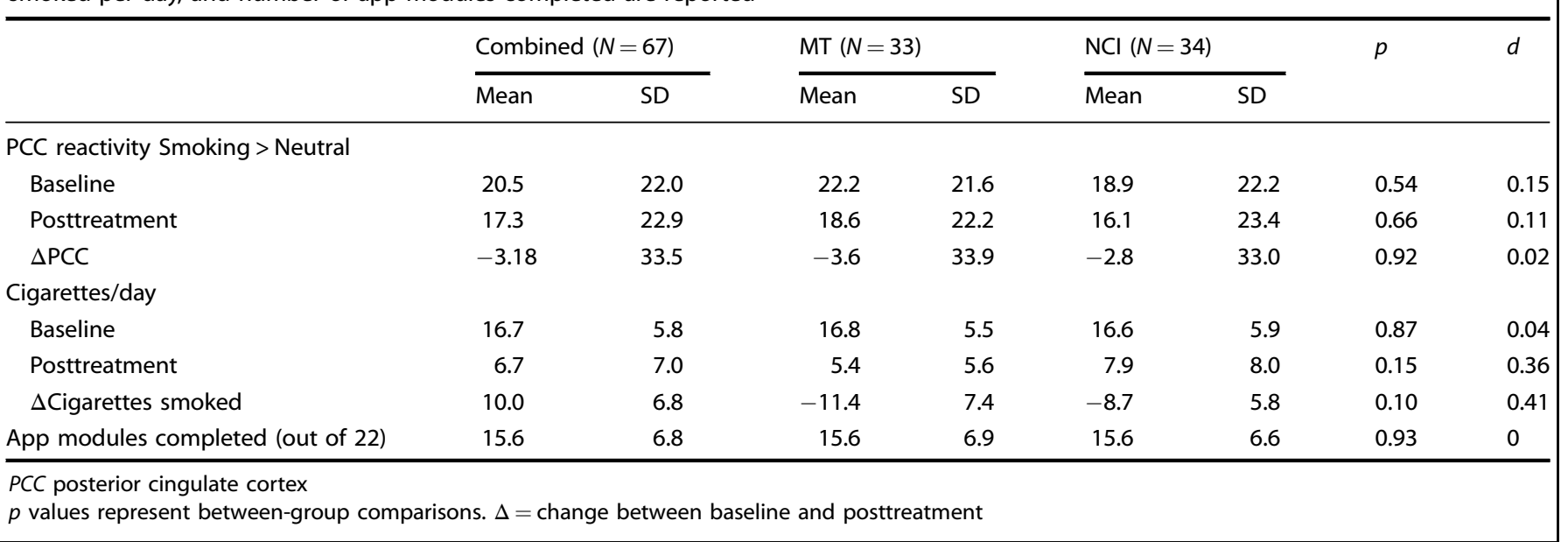

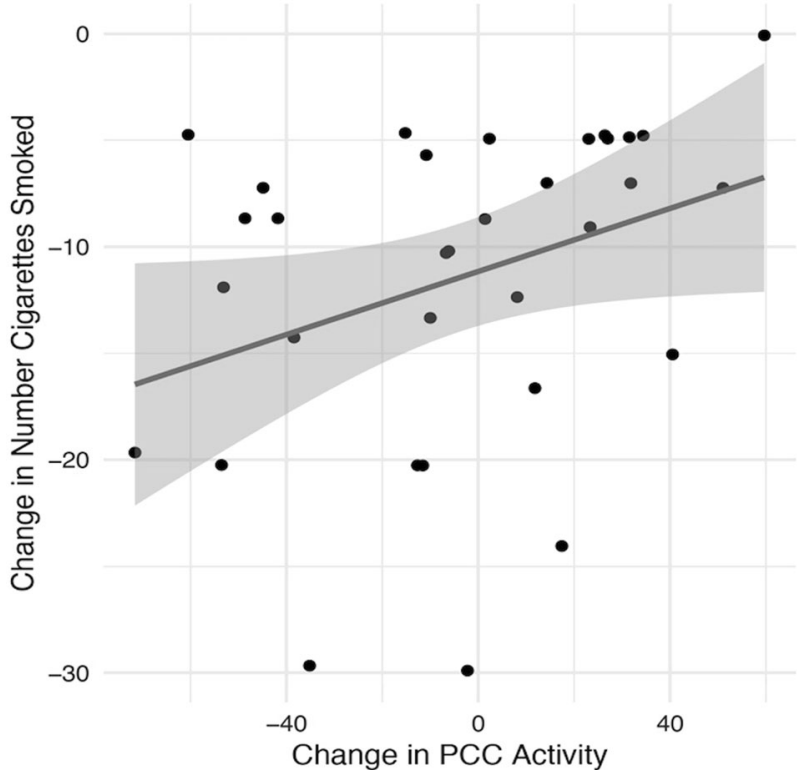

Fig. 3 Correlations between change in number of cigarettes smoked and change in PCC activity from baseline to post intervention $(\triangle P C C)$ in the Mindfulness Training group. PCC posterior cingulate cortex

Recent work has used brain-based phenotyping ("biotyping") of extreme individual deviations to improve diagnostic power and accuracy in psychiatric disorders, as well as gauge severity of substance use disorders $[14,32,33]$. In relation to the current work, individual variance in smoking cue-reactivity has been noted and in response, a call has been made to attend to such variance in guiding treatment $[42,43]$. For example, prior studies have found that greater baseline insula reactivity to smoking cues predicted medication-assisted outcomes [38, 44]. To our knowledge, the current study is the first to identify a behavioral "treatment biotype" for predicting clinical outcomes at the level of the individual that brings together consistent theoretical models of addiction (operant conditioning), treatment (MT) and associated brain mechanisms (PCC). From a treatment standpoint, these findings are important, as they suggest that heightened pretreatment PCC cue reactivity may identify individuals who are more responsive to specific interventions that target reducing PCC activity (e.g. MT). As a direct next step, novel methods based on baseline cue-reactivity can be developed for screening, prospectively selecting and personalizing treatment delivery to maximize the likelihood of success.

As relapse can be precipitated by many factors, cessation strategies that do not specifically target certain precipitants (e.g. cue-reactivity) may leave individuals vulnerable to the motivational effects of cues. This conjecture is supported by studies of medication-assisted cessation treatments such as nicotine replacement therapy (NRT), which has not demonstrated a specific effect on cue-reactivity [45]. Specifically, individuals with relatively greater smoking cue-reactivity prior to cessation aided by NRT were the most likely to relapse [7, 32, 38, 42-44, 46], indicating how treatments that do not effectively mitigate cue reactivity may leave individuals vulnerable to relapse. Thus, while smoking may be reduced in the short term with more broadly focused treatments (e.g. $\mathrm{NCl}$ ), cue-reactivity may contribute to relapse vulnerability when not coupled with a concurrent reduction of PCC reactivity to smoking cues. Future work will require assessing longer-term outcomes to determine the lasting impact of MT or other modalities that specifically target cue-reactivity to aid in cessation.

Our post-hoc analysis revealed an effect of sex such that the relationship between the reduction in PCC reactivity and smoking was noted only in female, but not male smokers, suggesting that MT may be particularly effective for women. This finding may be explained by work indicating that men and women smoke for different underlying reasons; men are more sensitive to the reinforcing effects of nicotine while women smoke to mitigate depression and negative affect [47]. Similar to the discussion regarding smokers having difficulty disengaging from cueinduced internal states, a hallmark of major depressive disorder (MDD) is the inability to shift out of internally focused, ruminative thinking [48], which has been linked with enhanced DMN and PCC reactivity during exposure to negative images [49]. These findings fit with the overarching role of the PCC as a brain region driving internal focus no matter the trigger. One possibility is that women are generally more likely to engage and stay stuck in such ruminative states, explaining the higher rates of depression [50] and the stronger link between MDD and smoking in women relative to men [47]. Collectively, the current and prior studies suggest that in comparison to men, women are more likely to smoke due to the engagement of PCC-mediated internal states, and are more likely to reduce smoking when cue-induced PCC reactivity is mitigated. In contrast to women, men are more responsive to traditional pharmacotherapy, which blunts the pharmacological influence of nicotine and withdrawal [51-53]. 
Thus, the current work suggests that MT may be an effective alternative targeting smoking relapse risk factors that are more relevant to women vs. men. This sex difference may also explain the nonsignificant difference when comparing the $\mathrm{MT}$ and $\mathrm{NCl}$ groups as a whole as there were only nine women in the $\mathrm{NCl}$ group. Future research is required to explore these sex-specific effects more fully.

While this study has a number of strengths and notable findings, including carefully controlling for treatment using an active control, accounting for engagement and expectancy, assuring adequate sample sizes, and minimizing risk of bias (Cochrane risk bias analysis demonstrated low risk in six of seven categories [54]), there are several limitations, including the short duration of follow-up for smoking outcomes and the specific focus on a single region of interest. Future studies are needed to determine broader neural networks and network connectivity changes that may be involved in mindfulness-based behavior change, and the long-term effects of app-delivered mindfulness training that links changes in brain activity to smoking cessation outcomes. Additionally, studies involving larger sample sizes are required to more thoroughly evaluate sex, including the impact of the menstrual cycle in women. While the current study did not consider hormonal variation in women, these findings indicate differences in male and female smokers, which is a critical first step to understanding the sex-specific effects of MT. Further, the current finding fits with the theoretical framework that men and women smoke for different reasons and mitigating a PCC-mediated internal focus is more strongly linked with a reduction in smoking in women. Irrespective of these limitations, the current work supports the hypothesis that mindfulness training reduces PCC reactivity to smoking cues in those who decrease smoking behavior regardless of sex, thus providing a neurobiological mechanism for its effectiveness, and potential biotype markers that can be assessed for personalization and optimization of treatment and resultant clinical outcomes.

\section{FUNDING AND DISCLOSURE}

This study was supported by the following National Center for Complementary and Integrative Health (NCCIH) grants: 1R61AT009337-02 and 1R61AT009337-02S. ACJ is supported by the National Institute on Drug Abuse grants: K02DA042987 and R01DA039135. JAB is the founder of MindSciences, the company that developed the mindfulness app used in this study. He owns stock in, serves as a non-compensated scientist for the company and has previously served on the board of directors. This financial interest has been disclosed to and is being managed by Brown University, in accordance with its Conflict of Interest and Conflict of Commitment policies. All other authors report no biomedical financial interests or potential conflicts of interest.

\section{ACKNOWLEDGEMENTS}

We would like to thank Brian Keefe and David Vaughan at ICF International for providing us with $\mathrm{NCl}$ QuitGuide app data. We would also like to thank Eileen Small for her review of and feedback on the manuscript.

\section{ADDITIONAL INFORMATION}

Supplementary Information accompanies this paper at (https://doi.org/10.1038/ s41386-019-0403-y).

Publisher's note: Springer Nature remains neutral with regard to jurisdictional claims in published maps and institutional affiliations.

\section{REFERENCES}

1. Centers for Disease Control and Prevention. Cigarette smoking among adults, United States. Morb Mortal Wkly Rep. 2002;51:642-7.
2. Benowitz NL. Neurobiology of nicotine addiction: implications for smoking cessation treatment. Am J Med. 2008;121:3-10.

3. Shiffman S, Paty JA, Gnys M, Kassel JA, Hickcox M. First lapses to smoking: withinsubjects analysis of real-time reports. J Consult Clin Psychol. 1996;64:366-79.

4. Drobes DJ, Tiffany ST. Induction of smoking urge through imaginal and in vivo procedures: physiological and self-report manifestations. J Abnorm Psychol. 1997; 106:15-25

5. Conklin CA, Vella EJ, Joyce CJ, Salkeld RP, Perkins KA, Parzynski CS. Examining the relationship between cue-induced craving and actual smoking. Exp Clin Psychopharmacol. 2015;23:90-96.

6. Ferguson SG, Shiffman S. The relevance and treatment of cue-induced cravings in tobacco dependence. J Subst Abus Treat. 2009;36:235-43.

7. Engelmann JM, Versace F, Robinson JD, Minnix JA, Lam CY, Cui Y, et al. Neural substrates of smoking cue reactivity: a meta-analysis of fMRI studies. Neuroimage. 2012;60:252-62.

8. Buckner RL, Andrews-Hanna JR, Schacter DL. The brain's default network: anatomy, function, and relevance to disease. Ann NY Acad Sci. 2008;1124:1-38.

9. Brewer JA, Garrison KA, Whitfield-Gabrieli S. What about the "self" is processed in the posterior cingulate cortex? Front Hum Neurosci. 2013;7:1-7.

10. Whitfield-Gabrieli S, Ford JM. Default mode network activity and connectivity in psychopathology. Annu Rev Clin Psychol. 2012;8:49-76.

11. Whitfield-Gabrieli S, Moran JM, Nieto-Castañón A, Triantafyllou C, Saxe R, Gabrieli JDE. Associations and dissociations between default and self-reference networks in the human brain. Neuroimage. 2011;55:225-32.

12. Mason MF, Norton MI, Van Horn JD, Wegner DM, Grafton ST, Macrae CN. Wandering minds: the default network and stimulus-independent thought. Science (80-). 2007. https://doi.org/10.1126/science.1131295.

13. Janes $A C$, Betts J, Jensen JE, Lukas SE. Dorsal anterior cingulate glutamate is associated with engagement of the default mode network during exposure to smoking cues. Drug Alcohol Depend. 2016;167:75-81.

14. Claus ED, Blaine SK, Filbey FM, Mayer AR, Hutchison KE. Association between nicotine dependence severity, BOLD response to smoking cues, and functional connectivity. Neuropsychopharmacology. 2013;38:2363-72.

15. Garavan H, Pankiewicz J, Bloom A, Cho JK, Sperry L, Ross TJ, et al. Cue-induced cocaine craving: neuroanatomical specificity for drug users and drug stimuli. Am J Psychiatry. 2000;157:1789-98.

16. Van Holst RJ, Van Den Brink W, Veltman DJ, Goudriaan AE. Brain imaging studies in pathological gambling. Curr Psychiatry Rep. 2010;12:418-25.

17. Jarraya B, Brugières $P$, Tani N, Hodel J, Grandjacques B, Fénelon G, et al. Disruption of cigarette smoking addiction after posterior cingulate damage. J Neurosurg. 2010;113:1219-21.

18. Garrison KA, Santoyo JF, Davis JH, Thornhill TA, Kerr CE, Brewer JA. Effortless awareness: using real time neurofeedback to investigate correlates of posterior cingulate cortex activity in meditators' self-report. Front Hum Neurosci. 2013;7:1-9.

19. Garrison KA, Scheinost D, Worhunsky PD, Elwafi HM, Thornhill TA, Thompson E, et al. Real-time fMRI links subjective experience with brain activity during focused attention. Neuroimage. 2013;81:110-8.

20. Brewer JA, Worhunsky PD, Gray JR, Tang Y-Y, Weber J, Kober H. Meditation experience is associated with differences in default mode network activity and connectivity. Proc Natl Acad Sci USA. 2011;108:20254-9.

21. Leech R, Sharp DJ. The role of the posterior cingulate cortex in cognition and disease. Brain. 2014;137:12-32.

22. Garrison KA, Zeffiro TA, Scheinost D, Constable RT, Brewer JA. Meditation leads to reduced default mode network activity beyond an active task. Cogn Affect Behav Neurosci. 2015;15:712-20.

23. Fox $K C R$, Dixon $M L$, Nijeboer $S$, Girn $M$, Floman JL, Lifshitz $M$, et al. Functional neuroanatomy of meditation: a review and meta-analysis of 78 functional neuroimaging investigations. Neurosci Biobehav Rev. 2016;65:208-28.

24. Brewer JA, Ruf A, Beccia AL, Essien Gl, Finn LM, Lutterveld Rvan, et al. Can mindfulness address maladaptive eating behaviors? Why traditional diet plans fail and how new mechanistic insights may lead to novel interventions. Front Psychol. 2018;9:1-11.

25. Gu X, Filbey F. A Bayesian observer model of drug craving. JAMA Psychiatry 2017;74:419.

26. Brewer JA, Elwafi H, Davis J. Craving to quit: psychological models and neurobiological mechanisms of mindfulness training as treatment for addictions. Psychol Addict Behav. 2013;27:366-79.

27. Creswell JD. Mindfulness interventions. Annu Rev Psychol. 2017;68:491-516.

28. Bowen S, Witkiewitz K, Clifasefi SL, Grow J, Chawla N, Hsu SH, et al. Relative efficacy of mindfulness-based relapse prevention, standard relapse prevention, and treatment as usual for substance use disorders. JAMA Psychiatry. 2014;71:547-56.

29. Garland EL, Manusov EG, Froeliger B, Kelly A, Williams JM, Howard MO. Mindfulness-oriented recovery enhancement for chronic pain and prescription 
opioid misuse: Results from an early-stage randomized controlled trial. J Consult Clin Psychol. 2014;82:448-59.

30. Brewer JA, Mallik S, Babuscio TA, Nich C, Johnson HE, Deleone CM, et al. Mindfulness training for smoking cessation: results from a randomized controlled trial. Drug Alcohol Depend. 2011;119:72-80.

31. Elwafi HM, Witkiewitz K, Mallik S IV, TAT, Brewer JA. Mindfulness training for smoking cessation: moderation of the relationship between craving and cigarette use. Drug Alcohol Depend. 2013;130:222-9.

32. Tamminga CA, Ivleva El. Using brain-based phenotyping to improve discovery in psychiatry. JAMA Psychiatry. 2018;75:1103.

33. Wolfers T, Doan NT, Kaufmann T, Alnæs D, Moberget T, Agartz I, et al. Mapping the heterogeneous phenotype of schizophrenia and bipolar disorder using normative models. JAMA Psychiatry. 2018;75:1146-55.

34. Biener L, Abrams DB. The contemplation ladder: validation of a measure of readiness to consider smoking cessation. Heal Psychol. 1991;10:360-5.

35. Garrison KA, Pal P, Rojiani R, Dallery J, O'Malley SS, Brewer JA. A randomized controlled trial of smartphone-based mindfulness training for smoking cessation: a study protocol. BMC Psychiatry. 2015;15:1-7.

36. Devilly GJ, Borkovec TD. Psychometric properties of the credibility/expectancy questionnaire. J Behav Ther Exp Psychiatry. 2000;31:73-86.

37. Janes AC, Farmer S, Peechatka AL, Frederick BDB, Lukas SE. Insula-dorsal anterior cingulate cortex coupling is associated with enhanced brain reactivity to smoking cues. Neuropsychopharmacology. 2015;40:1561-8.

38. Janes AC, Pizzagalli DA, Richardt S, Frederick B, de B, Chuzi S, et al. Brain reactivity to smoking cues prior to smoking cessation predicts ability to maintain tobacco abstinence. Biol Psychiatry. 2010;67:722-9.

39. Janes AC, Ross RS, Farmer S, Frederick BB, Nickerson LD, Lukas SE, et al. Memory retrieval of smoking-related images induce greater insula activation as revealed by an fMRI-based delayed matching to sample task. Addict Biol. 2015;20:349-56.

40. McClernon FJ, Kozink RV, Lutz AM, Rose JE. 24-h smoking abstinence potentiates fMRI-BOLD activation to smoking cues in cerebral cortex and dorsal striatum. Psychopharmacology (Berlin). 2009;204:25-35.

41. Janes AC, Frederick BD, Richardt S, Burbridge $C$, Merlo-Pich E, Renshaw PF, et al. Brain $\mathrm{fMRI}$ responses to smoking-related images prior to and during extended smoking abstinence. Exp Clin Psychopharmacol. 2009;17:365-73.
42. Hanlon CA, Hartwell KJ, Canterberry M, Li X, Owens M, LeMatty T, et al. Reduction of cue-induced craving through realtime neurofeedback in nicotine users: the role of region of interest selection and multiple visits. Psychiatry Res Neuroimaging. 2013;213:79-81.

43. McClernon FJ, Kozink RV, Rose JE. Individual differences in nicotine dependence, withdrawal symptoms, and sex predict transient fMRI-BOLD responses to smoking cues. Neuropsychopharmacology. 2008;33:2148-57.

44. Janes AC, Gilman JM, Radoman M, Pachas G, Fava M, Evins AE. Revisiting the role of the insula and smoking cue-reactivity in relapse: a replication and extension of neuroimaging findings. Drug Alcohol Depend. 2017;179:8-12.

45. Waters AJ, Shiffman S, Sayette MA, Paty JA, Gwaltney CJ, Balabanis MH. Cueprovoked craving and nicotine replacement therapy in smoking cessation. J Consult Clin Psychol. 2004;72:1136-43.

46. Hanlon CA, Jones EM, Li X, Hartwell KJ, Brady KT, George MS. Individual variability in the locus of prefrontal craving for nicotine: Implications for brain stimulation studies and treatments. Drug Alcohol Depend. 2012;125:239-43.

47. Benowitz NL, Hatsukami D. Gender differences in the pharmacology of nicotine addiction. Addict Biol. 1998;3:383-404.

48. Holtzheimer PE, Mayberg HS. Stuck in a rut: rethinking depression and its treatment. Trends Neurosci. 2011;34:1-9.

49. Kaiser RH, Andrews-Hanna JR, Wager TD, Pizzagalli DA. Large-scale network dysfunction in major depressive disorder. JAMA Psychiatry. 2015;72:603.

50. Kessler R, McGonagle K, Zhao S, Nelson C, Hughes M, Eshleman S, et al. Lifetime and 12-month prevalence of DSM-III-R psychiatric disorders in the United States: results from the National Comorbidity Survey. Arch Gen Psychiatry. 1994;51:8-19.

51. Cepeda-Benito A, Reynoso JT, Erath S. Meta-analysis of the efficacy of nicotine replacement therapy for smoking cessation: differences between men and women. J Consult Clin Psychol. 2004;72:712-22.

52. Smith PH, Bessette AJ, Weinberger AH, Sheffer CE, McKee SA. Sex/gender differences in smoking cessation: a review. Prev Med (Balt). 2016;92:135-40.

53. Perkins KA, Scott J. Sex differences in long-term smoking cessation rates due to nicotine patch. Nicotine Tob Res. 2008;10:1245-51.

54. Higgins JPT, Altman DG, Gotzsche PC, Juni $P$, Moher D, Oxman AD, et al. The Cochrane Collaboration's tool for assessing risk of bias in randomised trials. BMJ. 2011;343:1-9. 